\title{
The OPERA experiment and its contribution to neutrino physics
}

\author{
Giovanni De Lellis*广 \\ Università "Federico II" and INFN, Naples, Italy \\ E-mail: giovanni.de.lellis@cern.ch
}

\begin{abstract}
The OPERA experiment has been running with the CNGS neutrino beam from 2008 to 2012 and collected $1.8 \times 10^{20}$ protons on target. Five tau neutrino candidates were observed, with a background of 0.25 events, leading to the discovery of tau neutrino appearance in a muon neutrino beam, reported in 2015. The analysis of the emulsion films is now completed. We report preliminary results obtained with the analysis of the final sample. We also report a study on cosmic-ray annual modulation. We then give some final remarks including the near future plans of the Collaboration.
\end{abstract}

The 3rd International Symposium on "Quest for the Origin of Particles and the Universe" 5-7 January 2017

Nagoya University, Japan

\footnotetext{
* Speaker.

${ }^{\dagger}$ on behalf of the OPERA Collaboration.
} 


\section{Introduction}

In 1998, at the Neutrino Conference in Takayama, the Super-Kamiokande Collaboration reported the evidence for muon neutrino oscillation [1]. The measurement of the muon and electron atmospheric neutrino flux showed that electron neutrinos were consistent with the expectations while muon neutrinos showed a zenith angle dependent deficit. This deficit and its zenith angle dependence were the first evidence for neutrino oscillations. The muon neutrino disappearance firstly seen by Super-Kamiokande was later confirmed with artificial neutrino beams by the K2K experiment in Japan [2] and by the MINOS experiment in the USA [3],

In 1998 tau neutrinos had not been observed yet. Therefore it was not clear whether muon neutrinos were oscillating to tau or to a sterile neutrino. The OPERA experiment [4] was designed to prove that muon neutrinos were oscillating to tau neutrinos, through the direct observation of tau neutrino charged-current interactions in a muon neutrino beam. The construction at CERN of the CNGS (CERN to Gran Sasso) muon neutrino beam was approved in December 1999 and OPERA started its construction in the underground INFN Gran Sasso laboratory in 2003. The CNGS was designed as a high-energy and high-intensity muon neutrino beam with a small contamination of electron neutrinos (within 1\%) and of muon anti-neutrinos (2\% in terms of interactions). The long baseline of $730 \mathrm{~km}$ was appropriate to investigate the oscillations in the atmospheric neutrino sector. The detection of short-lived particles like the $\tau$ lepton required a micrometric accuracy. The $\tau$ lepton detection is achieved with a hibrid design, combining nuclear emulsions acting as tracking detectors with micrometric accuracy and electronic detectors providing the time stamp to the event, identifying the region where neutrinos interacted and measuring the muon charge and momentum.

The detector at Gran Sasso took data from 2008 to 2012, integrating $1.8 \times 10^{20}$ protons on target. The first tau neutrino candidate was reported in 2010 [5]: the candidate was showing a $\tau \rightarrow \rho v_{\tau}$ decay, with subsequent $\rho \rightarrow \pi^{0} \pi$ and $\pi^{0} \rightarrow \gamma \gamma$ decay chains. The second [6] and third [7] candidates were reported in 2013, respectively in the $\tau \rightarrow \pi \pi \pi v_{\tau}$ and $\tau \rightarrow \mu \bar{v}_{\mu} \nu_{\tau}$ decay channels. The forth candidate was reported in 2014 [8] while the discovery of $v_{\tau}$ appearance was achied in 2015 with the observation of a fifth tau neutrino candidate over an expected background of 0.25 events [9]. The charmed hadron production in muon neutrino interactions was used as a control sample to check the efficiency for the detection of the tau lepton, given the similar lifetime of charmed hadrons (about $10^{-12} \mathrm{~s}$ ). 50 events were observed while $54 \pm 4$ were expected. A good agreement was found also in the shape of the relevant kinematical and topological variables, like the angle in the transverse plane between the charmed hadron and the muon and the impact parameter of the decay daugther particles with respect to the primary neutrino interaction vertex [10]. The discovery of tau neutrino appearance was explicitely cited in the Scientific Background of the 2015 Nobel Prize in Physics.

\section{An event with three vertices}

After the discovery of tau neutrino appearance, an effort was paid to complete the scanning and analysis of all the bricks selected in the first two places of the probability map defined for each event by the electronic detector data. As a result, 7132 events were located and 6785 have the full decay search performed. While analysing this sample, a peculiar event was found. We 
report the preliminary results of the event analysis. This event shows a three vertex topology as illustrated in Figure 1 where several images of the emulsion films taken at different depths and with high magnification are superimposed. At the primary vertex denoted as I, three tracks are attached, denoted as 2, 4 and 5; after a flight length of $103 \mu \mathrm{m}$, the secondary vertex denoted as II shows two tracks attached, denoted as 1 and 3 while the track 4 is the parent of a kink topology. The kink angle is $90 \mathrm{mrad}$ and the daughter momentum, measured with the multiple Coulomb scattering [11], is $2.7 \mathrm{GeV} / c$, giving a transverse momentum of $240 \mathrm{MeV} / c$. At the kink vertex there is a $\gamma$ attached inducing a large electromagnetic shower inside the brick. At the 2-prong vertex (II) the two charged particles show an invariant mass of $(1.8 \pm 0.5) \mathrm{GeV} / c^{2}$. The short flight lengths, the high invariant mass and transverse momentum measured are all indicators for the production of two heavy particles. This peculiar topology can be due either to a charmed hadron production in tau neutrino interactions or to a neutral current interaction with a $Z$ boson-gluon fusion mechanism giving a pair of charmed quarks. A dedicated Monte Carlo simulation has been carried out to include these processes in the event generator and to estimate their expected yield after the topological selection. For the same selection other background sources were considered, the largest being a single charm production in muon neutrino interactions with the misidentified muon at the primary vertex and with a pion hadronic interaction that mimics a secondary vertex.

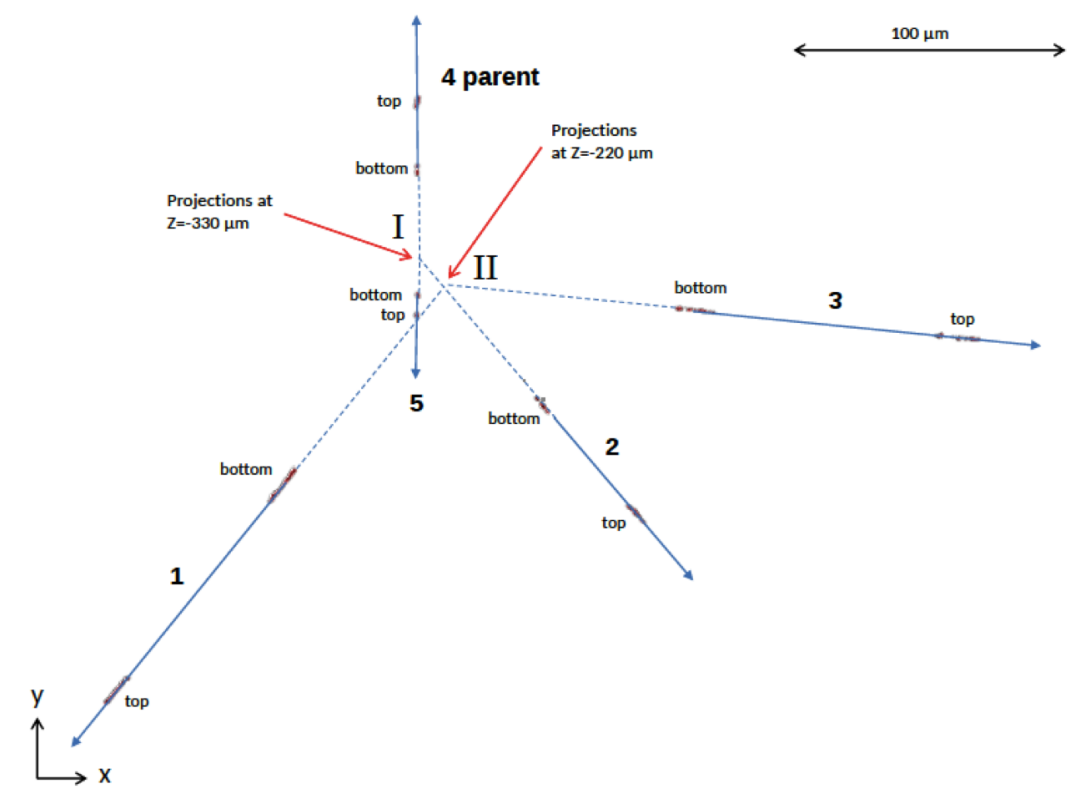

Figure 1: Superimposed images of the vertex region at different depths in the emulsion layer. The primary vertex (I), a secondary vertex (II) and the parent particle (4) of a single-prong decay vertex are visible.

Given the transverse momentum lower than $300 \mathrm{MeV} / c$, the event does not pass the standard selection for tau neutrino candidates. So a dedicated analysis has been carried out, based on the distribution of kinematical variables. Events are selected regardless of the multiplicity at the primary, by requiring that no muon nor electron are identified at the primary vertex, two secondary vertices are reconstructed, one with a single prong and another one with two prongs, and no nu- 
clear fragment is visible at the secondary vertices. Figure 2 shows different processes producing the observed topology and their yield after the topological selection. A total yield of 0.1 events is expected.

\begin{tabular}{l|c|c} 
Sample & Muon misidentified & Expected events $\left(10^{-3}\right)$ \\
\hline$\nu_{\tau} \mathrm{CC}+$ charm & 45 \\
$\nu_{\mu} \mathrm{CC}+$ charm $+h_{\text {int }}$ & yes & 21 \\
$\nu_{\mu} \mathrm{NC}+c \bar{c}$ & & 13 \\
$\nu_{\tau} \mathrm{CC}+h_{\text {int }}$ & yes & 9 \\
$\nu_{\mu} \mathrm{CC}+2 h_{\text {int }}$ & & 4 \\
$\nu_{\mu} \mathrm{NC}+2 h_{\mathrm{int}}$ & & 4 \\
\hline Total & & 100
\end{tabular}

Figure 2: Different processes giving the observed topology with the corresponding yield after the topological selection.

For the multivariate analysis, 12 kinematical variables are used, e.g. the transverse momentum at the decay vertex, the daughter momentum and the angle between the two parents in the transverse plane. The latter variable provides the best separation between charm production in tau neutrino interactions and double charm production in neutral current muon neutrino interactions.
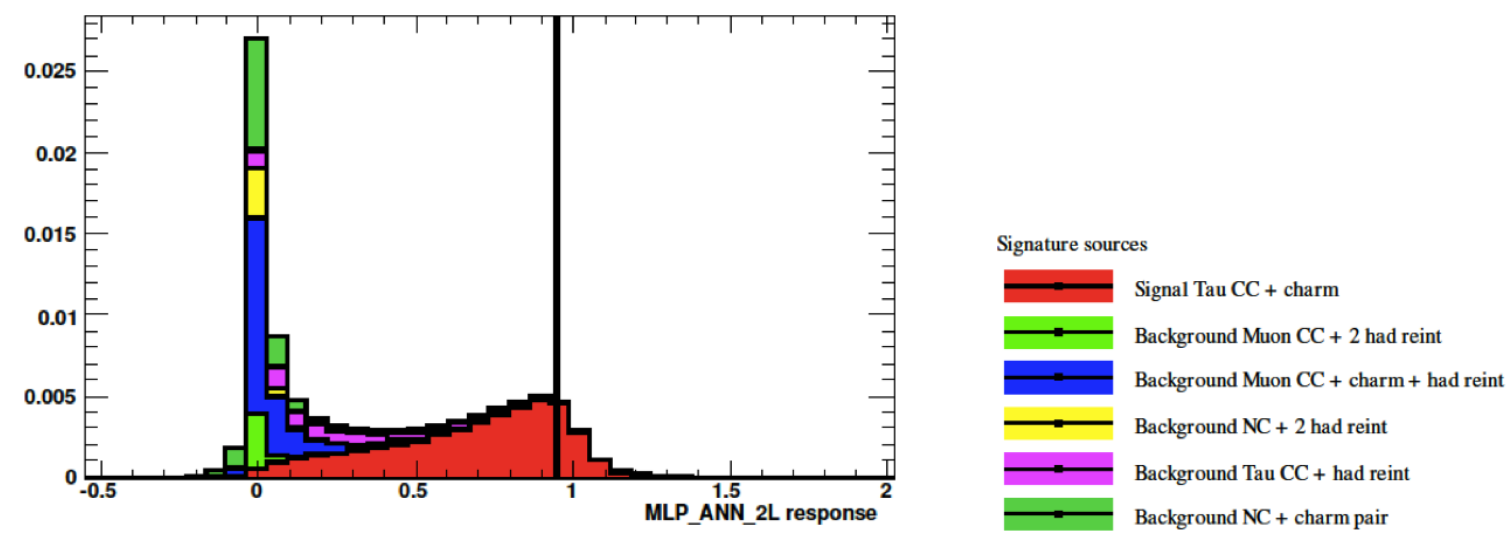

Figure 3: Distribution of the ANN output variable. The weighted contribution of each source listed in figure 2 is shown with a different colour. The vertical black line corresponds to the ANN output value for the event.

In order to discriminate among the different processes, the Artificial Neural Networks (ANN) method is used, The output variable of this method is shown in Figure 3 where the vertical black line corresponds to the ANN output value for the event. According to the multivariate analysis, the event can be classified as a tau neutrino charged-current interaction with charmed hadron production. The significance of this observation is evaluated using RooFit/RooStats libraries provided by the ROOT framework. The observable of the model is the ANN output variable $x$ whose distribution, normalised to the expected event yields, is shown in Fig. 3. The test statistics used for the significance evaluation is the profile likelihood ratio. A sample of simulated pseudo-experiments 
is generated according to the background only hypothesis, obtaining the test statistic distribution shown in Fig. 4. Under the background only hypothesis, the probability of data being less likely than the observed event is $2.6 \times 10^{-4}$, corresponding to the evidence for the tau neutrino interaction with charmed hadron production with a significance of $3.5 \sigma$.

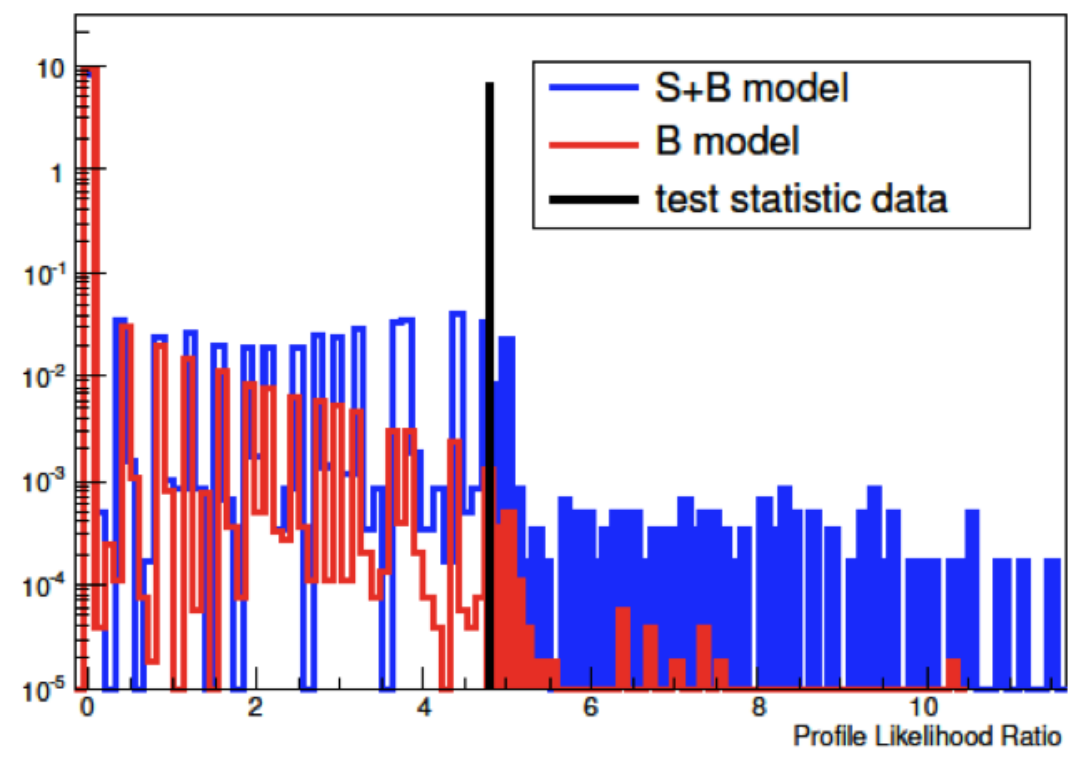

Figure 4: Distribution of the test statistic obtained with pseudo-experiments under the background only (B model) and signal plus background ( $\mathrm{S}+\mathrm{B}$ model) hypotheses.

\section{Cosmic-ray annual modulation}

Muons observed underground come mostly from the the decay of pions and kaons produced by the interaction of primary cosmic-rays with the nuclei of the upper atmosphere. Only most energetic muons can be observed in underground detectors, with an energy threshold of about 1.8 $\mathrm{TeV}$ for the Gran Sasso laboratory, below $3800 \mathrm{~m}$ of rock equivalent. In the upper atmosphere, above $15 \mathrm{~km}$, when the temperature increases, the density decreases thus increasing the mean free path of hadrons, hence yielding a higher pion decay rate and consequently a higher muon rate. Therefore, the flux of cosmic muons detected deep underground shows seasonal time variations related to the air density fluctuations. This effect was studied by several underground experiments including MACRO [12], LVD [13] and Borexino [14] at LNGS. The muon rate can therefore be written as

$$
I_{\mu}(t)=I_{\mu}^{0}+\Delta I_{\mu}=I_{\mu}^{0}+\delta I_{\mu} \cos \left[\frac{2 \pi}{T}\left(t-t_{0}\right)\right]
$$

where $T$ is the period and $t_{0}$ the phase of the modulation. We report here the preliminary results obtained with the analysis of the data collected in the runs from 2010 to 2012. The top plot of Figure 5 shows the cosmic muon signal as a function of time while the bottom one shows the variation of the effective temperature $T_{\text {eff }}$. The effective temperature is defined as the weighted 
average of the atmospheric temperature

$$
T_{\mathrm{eff}}=\frac{\int_{0}^{\infty} T(x) W(x) d x}{\int_{0}^{\infty} W(x) d x}
$$

where the weights $W$ are heavier in the upper layers of the atmosphere where higher energy muons orginate from. The curves show the sinusoidal fit to the data. In particular the period is well consistent with one year. When all three years are folded into one year, as shown in the left plot of Figure 6 , the phase gives a value $t_{0}=195$ days, corresponding to July $14^{\text {th }}$.
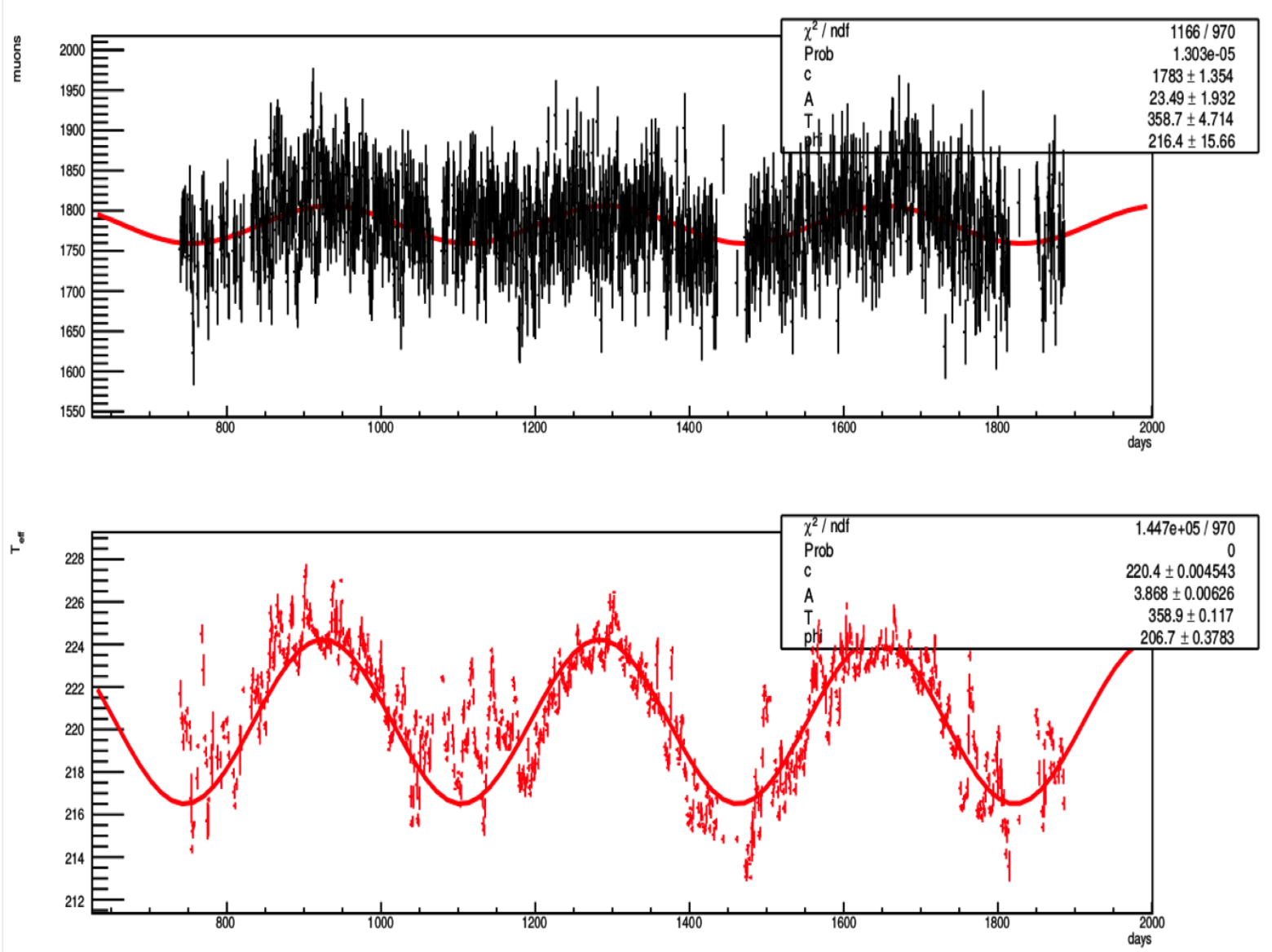

Figure 5: Upper plot: cosmic muon signal as a function of time. Bottom plot: effective temperature variation. The curves show the sinusoidal fit to the data.

The effective temperature coefficient $\alpha_{T}$ defined by the following relationship

$$
\frac{\Delta I_{\mu}}{I_{\mu}^{0}}=\alpha_{T} \frac{\Delta T_{\text {eff }}}{T_{\text {eff }}}
$$

quantifies the correlation effect between the muon rate and the effective temperature. A preliminary result for $\alpha_{T}$ is given by the fit performed in the right plot of Figure 6, yielding $\alpha_{T}=0.93 \pm 0.06$. This value is consistent with the measurement done with the Borexino detector [14]. 

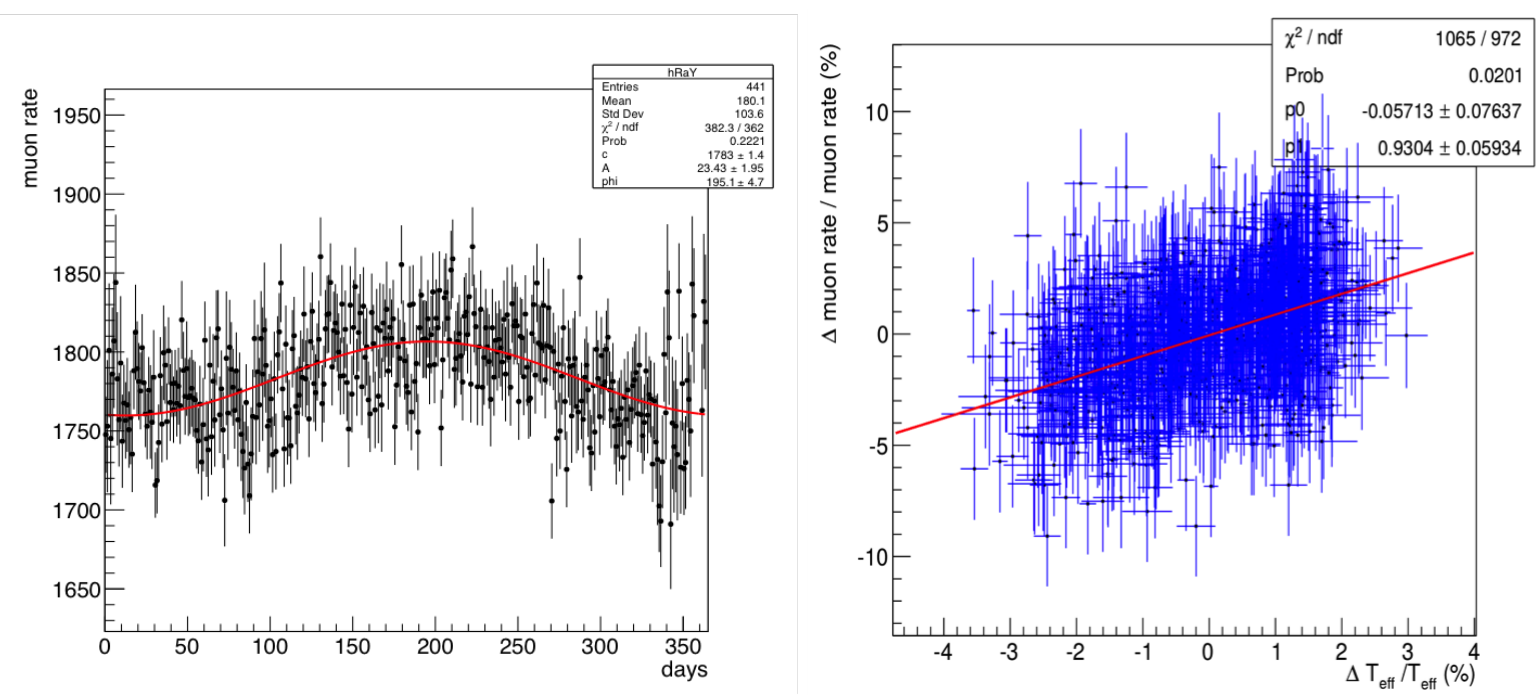

Figure 6: Left: cosmic muon rates for the three years folded onto a period of one year with the sinusoidal fit to the data. Right: $\Delta I_{\mu} / I_{\mu}^{0}$ versus $\Delta T_{\text {eff }} / T_{\text {eff }}^{0}$. Each point corresponds to one day of data.

\section{Conclusions and perspectives}

The OPERA Collaboration has concluded the scanning and analysis of all the events selected for the oscillation search. A new strategy is being applied: given the good description of the data reported in several papers, the Collaboration has decided to widen the selection cuts, hence collecting more candidate events. The selection cuts will be looser while keeping the same level of detail in the event analysis, including the track follow down procedure, meant for a highly efficient particle identification. This will lead to a less favourable signal to noise ratio that could be compensated by the exploitation of the event kinematical features with a multivariate approach. The statistical gain will reduce the uncertainty on the measurement of the oscillation parameters. For instance, the $\Delta m^{2}$ of the atmospheric sector could be meausured with a $20 \%$ accuracy for the first time in appearance mode. Preliminary results of this analysis will be reported at the Winter Conferences this year. The event reported in Section 2 is one of the candidates found in the enlarged data sample. OPERA will also exploit the unique feature of being able to identify all three neutrino flavours, combining electron and tau appearance with muon neutrino disappearance to constrain the parameters of the neutrino oscillation.

The OPERA Collaboration has also conducted a dedicated R\&D finalised to the improvement of the performance in the scanning systems, leading to an analysis speed almost one order of magnitude higher than five years ago $[15,16]$. This is paving the way for the application of nuclear emulsions to several experiments in different fields.

\section{References}

[1] Y. Fukuda et al., Super-Kamiokande Collaboration, Evidence for oscillation of atmospheric neutrinos, in Phys. Rev. Lett. 81 (1998) 1562-1567 
[2] E. Aliu et al., Evidence for muon neutrino oscillation in an accelerator-based experiment, Phys. Rev. Lett. 94 (2005) 081802

[3] D.G. Michael et al., Observation of muon neutrino disappearance with the MINOS detectors and the NuMI neutrino beam, Phys. Rev. Lett. 97 (2006) 191801.

[4] R. Acquafredda et al., The OPERA experiment in the CERN to Gran Sasso neutrino beam, JINST 4 (2009) P04018.

[5] N. Agafonova et al., Observation of a first $v_{\tau}$ candidate in the OPERA experiment in the CNGS beam, Phys. Lett. B691 (2010) 138.

[6] N. Agafonova et al., New results on $v_{\mu} \rightarrow v_{\tau}$ appearance with the OPERA experiment in the CNGS beam, JHEP 1311 (2013) 036.

[7] N. Agafonova et al., Evidence for $v_{\mu} \rightarrow v_{\tau}$ appearance in the CNGS neutrino beam with the OPERA experiment, Phys.Rev. D89 (2014) no.5, 051102

[8] N. Agafonova et al., Observation of tau neutrino appearance in the CNGS beam with the OPERA experiment OPERA Collaboration, PTEP 2014 (2014) no.10, 101C01

[9] N. Agafonova et al., Discovery of $\tau$ Neutrino Appearance in the CNGS Neutrino Beam with the OPERA Experiment OPERA Collaboration, Phys.Rev.Lett. 115 (2015) no.12, 121802

[10] N. Agafonova et al., Procedure for short-lived particle detection in the OPERA experiment and its application to charm decays, Eur. Phys. J. C74 (2014) 2986.

[11] G.De Lellis et al., Momentum measurement by the angular method in the Emulsion Cloud Chamber, Nucl. Instrum. Meth. A512 (2003) 539-545.

[12] M. Ambrosio et al., Seasonal variations in the underground muon intensity as seen by MACRO, Astroparticle Physics 7 (1997) 109.

[13] M. Selvi for the LVD Collaboration, Analysis of the seasonal modulation of the cosmic muon flux in the LVD detector during 2001-2008, XXXI International Comsci Ray Conference, July 7-15, Lodz, Poland (2009).

[14] G. Bellini et al., Cosmic-muon flux and annual modulation in Borexino at $3800 \mathrm{~m}$ water-equivalent depth, JCAP 1205 (2012) 015.

[15] A. Alexandrov et al., A new fast scanning system for the measurement of large angle tracks in nuclear emulsions, JINST 10 (2015) no.11, P11006.

[16] A. Alexandrov et al., A new generation scanning system for the high-speed analysis of nuclear emulsions, JINST 11 (2016) no.06, P06002. 